\title{
BMJ Open Evaluating the short-term and long-term effects of an internet-based aural rehabilitation programme for hearing aid users in general clinical practice: a randomised controlled trial
}

\author{
Milijana Malmberg, ${ }^{1,2}$ Thomas Lunner, ${ }^{3,4}$ Kim Kähäri, ${ }^{2}$ Gerhard Andersson ${ }^{3,5}$
}

To cite: Malmberg M, Lunner T, Kähäri K, et al. Evaluating the short-term and long-term effects of an internet-based aural rehabilitation programme for hearing aid users in general clinical practice: a randomised controlled trial. BMJ Open 2017;0:e013047. doi:10.1136/ bmjopen-2016-013047

- Prepublication history and additional material are available. To view these files please visit the journal online (http://dx.doi.org/ 10.1136/ bmjopen-2016-013047).

Received 16 June 2016 Accepted 21 March 2017

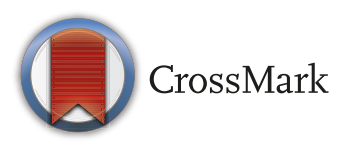

${ }^{1}$ Hearing Organization, Habilitation and Health, Region Västra Götaland, Sweden ${ }^{2}$ Department of Health and Rehabilitation, Institute of Neuroscience and Physiology, Sahlgrenska Academy, University of Gothenburg, Göteborg, Sweden

${ }^{3}$ Department of Behavioral Sciences and Learning, Swedish Institute for Disability Research, Linköping University, Linköping, Sweden

${ }^{4}$ Eriksholm Research Centre, Oticon A/S Snekkersten, Snekkersten, Denmark ${ }^{5}$ Department of Clinical Neuroscience, Karolinska Institutet, Stockholm, Sweden

Correspondence to Milijana Malmberg; milijana.lundberg.malmberg@ vgregion.se

\section{ABSTRACT}

Objective Guided internet-based intervention beyond hearing aid $(\mathrm{HA})$ fitting has been shown to be efficacious in randomised controlled trials (RCTs). However, internet interventions have rarely been applied clinically as a part of regular aural rehabilitation (AR). Our aim was to evaluate the effectiveness of internet-based AR for HA users from a clinical population.

Outcome measures The Hearing Handicap Inventory for the Elderly (HHIE) was used as the primary outcome measure, and the Communication Strategies Scale (CSS) and the Hospital Anxiety and Depression Scale were used as secondary outcome measures. All questionnaires were administered before and directly after the intervention and at 6 months postintervention.

Methods We used a parallel group design (RCT). The data were collected in 2013-2014 at three different clinics. Seventy-four HA users were randomly assigned to receive either full internet-based $A R$ (intervention group, $n=37$ ) or one element of the internet-based AR (control group, $n=37$ ).

Results Data were analysed following the intention-to-treat principle. Each group showed improved HHIE scores over time and did not differ significantly from each other. The intervention group showed significantly greater improvement compared with the control group for the CSS total and the non-verbal subscale scores. The intervention group and control group were also subdivided into two age groups: 20 59 years and $60-80$ years. Significantly better improvement on the CSS total and non-verbal subscale scores was found in the older group compared with the younger participants. Conclusions This study indicates that participants in an internet-based intervention applied in general clinical practice showed improved self-reported communication skills compared with a control group. Receiving a full intervention was not more effective in improving selfreported hearing problems than receiving just one element of the internet-based intervention.

Trial registration number This trial is registered at ClinicalTrals.gov, NCT01837550; results.

\section{INTRODUCTION}

Hearing impairment influences communication in people's daily life. In agreement with the International Classification of
Strengths and limitations of this study

This is one of the first randomised controlled trials in Sweden to implement internet-based rehabilitation beyond conventional hearing aid fitting in a general clinical practice.

- The recruitment process used in the clinical trial will provide indications of the types of hearing aid users who are interested in this type of intervention.

- One limitation of this study is that the control group received an active intervention.

- Another limitation of this study is that the control group received only one of the four elements of the programme, overlooking the relative benefit that any other element alone/combination of elements might have as compared with the full intervention.

Functioning, Disability and Health, ${ }^{1}$ the objective of aural rehabilitation (AR) is to promote social participation for people with hearing impairment. Addressing this objective includes fitting the client with hearing aids (HA), educating him or her about the condition and providing perceptual training and counselling ${ }^{2}$. To improve communication for people with hearing impairment, researchers recommend combining group AR with HA use. ${ }^{3-6}$ This combination has been shown to be more cost-effective than HA use alone. ${ }^{7}$ However, despite the recommendations, the most common approach is the use of HAs alone. ${ }^{8}$ This discrepancy could be explained by clinicians' lack of time and the difficulties of scheduling comprehensive AR in addition to HA fitting. ${ }^{9}$ Moreover, HA users with stressful life situations may have very limited time to spend on travelling to participate in rehabilitation courses offered by the clinic. Also, many HA users experience communication difficulties despite today's HA technology. This could cause patients 
to stop using their HAs, ${ }^{10}$ which can lead to withdrawal from and/or avoidance of interpersonal interactions or involvement in community life. A review of the literature showed that HA users' self-perceived hearing difficulties can affect help-seeking, HA uptake, HA use and satisfaction. ${ }^{11}$ Although combining group AR with HA use can be beneficial, the overall availability of and adherence to communication programmes are still low. ${ }^{12}$

Several studies have suggested that AR could be provided without in-person meetings, ${ }^{13-20}$ for example by providing educational programmes using telephone/ internet-based AR. A recent systematic review indicated that such resources show benefits such as increased access to care, cost-effectiveness and improved quality of care in terms of user satisfaction. ${ }^{20}$ Further on, internet use is increasing among people with hearing impairment, which encourages including the internet for AR in future research. ${ }^{21-23}$ There is evidence to suggest that learning and educational support delivered via the internet could support first-time HA users in clinical practice.${ }^{19}$ However, the effectiveness of clinical use of the internet for AR is sparsely examined.

Our research group designed a randomised controlled trial (RCT) of internet-based $\mathrm{AR}^{18}$. The results showed significantly increased activity and participation in the intervention group by using the internet to provide $\mathrm{AR}$ in addition to HA fitting, while the control group did not improve. The study provided proof of concept that AR beyond HA fitting could be performed over the internet. ${ }^{1618}$ However, participants were recruited through advertisements and articles in Swedish national daily newspapers and on the internet, and the study did not indicate whether internet-based interventions could be feasible if strictly administered in a clinical setting. Nonetheless, we chose to use this same RCT design ${ }^{18}$ as described above and supplement the trial with telephone support, and then implement the trial in a clinical setting at a later time. Our earlier research showed promising results for telephone-supported AR for HA users in general clinical practice (GCP). ${ }^{17}$ A study of self-help treatment for tinnitus in a clinical setting showed significant improvements pretreatment to post-treatment and at follow-up when internet-based treatments were used, indicating that self-help treatment can be transferred to the clinic. ${ }^{24}$ Studies in other research fields, such as panic disorders, have shown that guided internet-based therapy is efficacious and effective when delivered as part of routine psychiatric care. ${ }^{25}$

The first aim of this study was to evaluate whether internet-based AR for HA users will be effective in GCP. Our assumption was that the internet-based AR programme would reduce residual hearing problems among HA users and improve the participants' communication strategies and psychosocial well-being, while participating in the control group would not. The intervention groups' improvements post-treatment are expected to be maintained when assessed 6 months post-treatment. The second aim of the study was to analyse the effect of internet-based
AR in GCP among two age-groups: $20-59$ years and $60-80$ years. Our hypothesis was that the 20-59years age group may be more receptive to internet-based AR because of their presumably greater digital literacy skills, ${ }^{26}$ compared with those who are in the $60-80$ years age group.

\section{METHODS}

The Consolidated Standards of Reporting Trials checklist was followed when reporting the abstract, designing the study and analysing and interpreting the results. ${ }^{27}{ }^{28} \mathrm{~A}$ flow chart of the study procedure is presented in figure 1 . The trial is registered at ClinicalTrals.gov, NCT01837550.

\section{Recruitment and selection}

The eligibility criteria targeted the most common patient category at three clinics within the Hearing Organization, Södra Älvsborg, Sweden: patients who were 20-80 years old and who had conductive or sensorineural binaural hearing loss of 20-60Decibel Hearing Level (dB HL) pure-tone average $(500,1000$ and $2000 \mathrm{~Hz})$. Additional eligibility criteria included patients who had completed an HA fitting 3 months before the study began (regardless of HA manufacturer or model), who had an Hearing Handicap Inventory for the Elderly (HHIE) score $\geq 20$ points, ${ }^{29}$ indicative of some residual hearing problems, and who gave their informed consent to participate. The study was conducted in 2013-2014. There was no difference in the three clinics in terms of patients and general procedures. The recruitment process was conducted in two sets, one for participants aged 20-59 years and one for those aged $60-80$ years. All potential participants who fulfilled the criteria for age, hearing loss and HA fitting received a recruitment letter that contained information about the study's purpose and structure, and stressed that the participants' privacy would be protected and that participation was voluntary. The participants were prepared to allocate 1.5-2.0 hours each week to participate in the study and were informed that they would be placed into one of two groups. The participants were asked to visit the website www.iterapi.se/sites/hornet to read more about the study and to initiate participation.

The first step of the participation required registering at the website and completing a screening form. Participants who completed this first step $(n=108)$ were called by the project leader for a telephone interview to assess their eligibility; of these, 104 agreed to participate in the study. The next step was for the participants to return a signed consent form to the project leader and to complete four standardised questionnaires (see 'Outcome measures' below). Consequently, 74 participants were included in the study, as seen in figure 1.

The study was an RCT with a parallel group design and a simple randomisation procedure through the recruitment process that was conducted in two sets. The 74 participants were randomly assigned to either an intervention group (group 1) or a control group (group 2) according to a computer-generated list of random numbers. An 


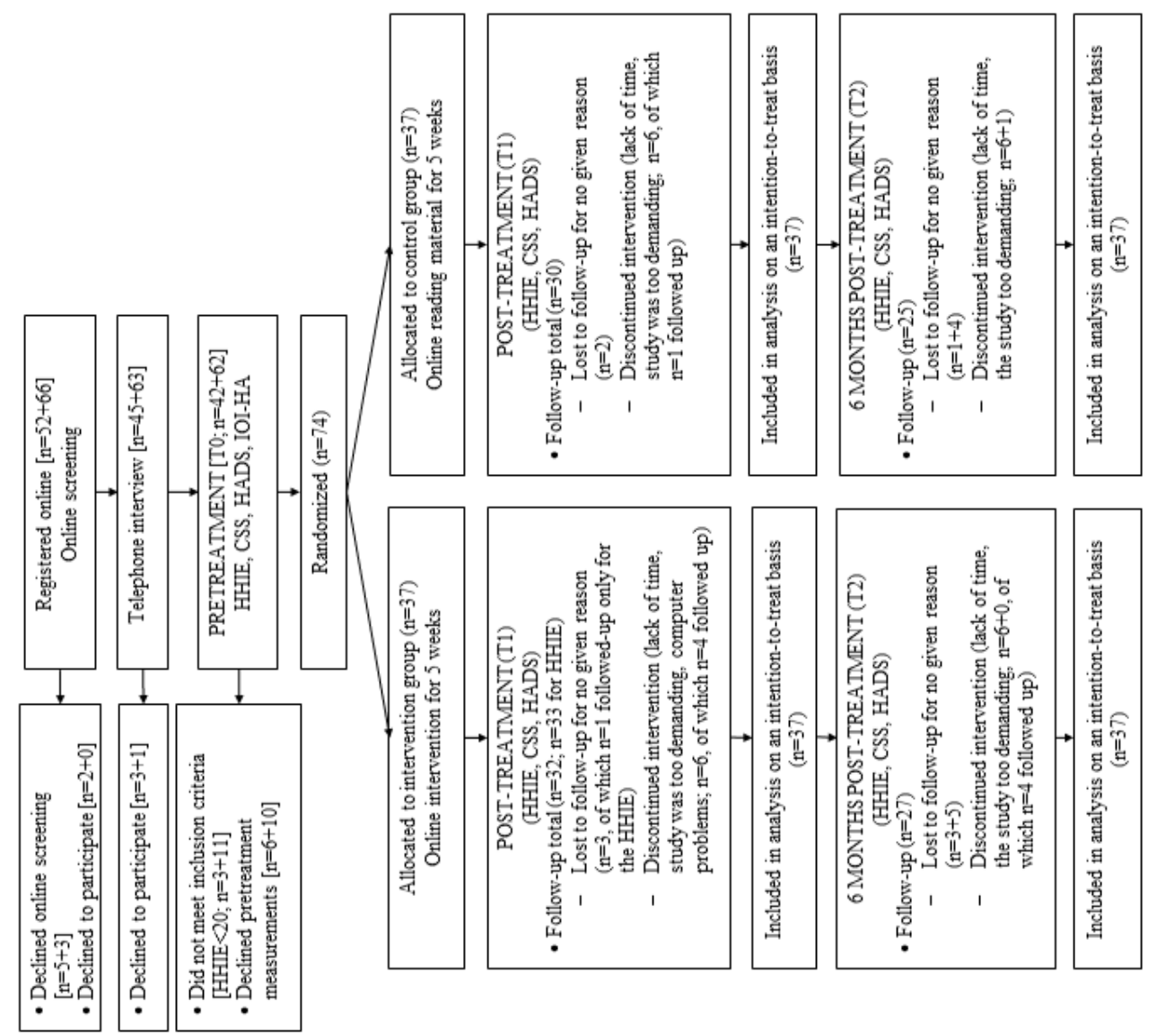

인

$\stackrel{1}{ \pm}$

○苋

흐음

๑

ㄷํ음

ฮิ ธิ

จั

워

II $\frac{x}{2}$

क

的至

舟

$+\infty$

il

c)

क .

突

वृ.

용

O \&

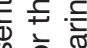

के के Ф

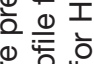

$\frac{\bar{\alpha}}{0.0}$

is 궁

응 웅

은

离 突 ह

है

언

눙

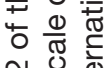

我心

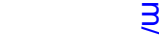

这

过恋

당

屯․ 을 흥

흥. 임

흘

गै के

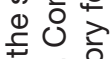

항

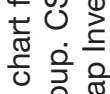

ว 흫웧

은 웜

$\pi I$
$-\quad 0$

잃

훈웜 
Table 1 Demographic and clinical characteristics of the participants

\begin{tabular}{|c|c|c|}
\hline & Intervention group $(\mathrm{n}=\mathbf{3 7})$ & Control group $(n=37)$ \\
\hline 20-59years age group, $\mathrm{n}(\%)$ & $17(46)$ & $16(43)$ \\
\hline 60-80years age group, $\mathrm{n}(\%)$ & $20(54)$ & $21(57)$ \\
\hline Age, years (range $31-80$ years) & $61.8(11.9)$ & $62.1(11.4)$ \\
\hline 20-59years age group & $50.9(7.2)$ & $52.3(9.1)$ \\
\hline $60-80$ years age group & $71.1(5.4)$ & $69.6(5.9)$ \\
\hline \multicolumn{3}{|l|}{ Gender, n (\%) } \\
\hline Men & $24(64.9)$ & $20(54.1)$ \\
\hline Woman & $13(35.1)$ & $17(45.9)$ \\
\hline \multicolumn{3}{|l|}{ Pure-tone average ( $\mathrm{dB} \mathrm{HL}$ ) } \\
\hline Right ear & $37.5(11.3)$ & $38.0(8.6)$ \\
\hline Left ear & $37.8(10.5)$ & $36.5(8.5)$ \\
\hline \multicolumn{3}{|l|}{$\mathrm{HA}, \mathrm{n}(\%)$} \\
\hline Binaural & $28(75.7)$ & $31(83.8)$ \\
\hline Monaural & $9(24.3)$ & $6(16.2)$ \\
\hline \multicolumn{3}{|l|}{ Duration of $\mathrm{HA}$ use } \\
\hline Years (range $0.5-55$ years) & $7.5(9.6)$ & $7.4(6.3)$ \\
\hline Median $(\mathrm{Q} 1 / \mathrm{Q} 3)^{\star}$ & $5.0(1.5 / 10.5)$ & $6.0(2.3 / 11.3)$ \\
\hline Computer experience†, n (\%) & $37(100)$ & $37(100)$ \\
\hline Computer access, n (\%) & 37 (100) & $37(100)$ \\
\hline Able to have a telephone conversation without $\mathrm{HA} / \mathrm{s} ?, \mathrm{n}(\%)$ & $32(86.5)$ & $35(94.6)$ \\
\hline \multicolumn{3}{|l|}{ IOI-HA } \\
\hline 1. Daily use & $4.1(1.1)$ & $4.4(1.0)$ \\
\hline 2. Benefit & $3.8(0.9)$ & $4.1(0.9)$ \\
\hline 3. Remaining activity limitation & $3.1(0.8)$ & $3.1(0.8)$ \\
\hline 4. Satisfaction & $4.1(1.1)$ & $4.4(0.8)$ \\
\hline 5. Remaining participation restriction & $3.8(1.1)$ & $4.1(0.8)$ \\
\hline 6. Impact on environment & $3.6(0.9)$ & $3.9(0.9)$ \\
\hline 7. Quality of life & $3.6(0.8)$ & $3.6(1.0)$ \\
\hline
\end{tabular}

The data are reported as means (SD) unless stated otherwise.

${ }^{*} \mathrm{Q} 1=$ the first quartile, $\mathrm{Q} 3=$ the third quartile.

†Familiar with: able to log in, print information, complete a questionnaire on a website, and read and send e-mail. HA, hearing aid; dB HL, Decibel Hearing Level; IOI-HA, International Outcome Inventory for Hearing Aids.

independent audiologist at the clinic (not involved in the recruitment) generated the random allocation sequence using a computer software program and assigned the participants to different groups. The independent audiologist reported the allocation schedule to the project leader, who then enrolled the participants. The assigned participants were told which group they were allocated to (1 or 2) but were not informed whether the group was the intervention group or the control group. Thirty-seven participants were included in the intervention group, and 37 participants were included in the control group, as shown in table 1 . No significant differences were found between the groups regarding the background variables age, age group, gender and hearing loss.

The study was reviewed and approved by the regional ethical review board in Gothenburg, Sweden. The study website was programmed using Java Script, and information was available in hypertext markup language format.

\section{Outcome measures}

The HHIE $^{29}$ was the primary outcome measure. The HHIE includes two subscales: the social subscale comprises 12 questions addressing the social effects of hearing loss, and the emotional subscale comprises 13 questions addressing the emotional effects of hearing loss. Higher scores reflect a higher self-reported hearing problem.

The Communication Strategies Scale (CSS, from the Communication Profile for the Hearing Impaired ${ }^{30}$ ) and the Hospital Anxiety and Depression Scale (HADS) ${ }^{31}$ were used as secondary outcome measures. The CSS includes three subscales (maladaptive behaviours, verbal strategies and non-verbal strategies) and is designed to analyse participants' 
behaviour in various communication situations. The maladaptive behaviours subscale includes nine questions that analyse strategies that hinder communication. Verbal strategies and non-verbal strategies address 16 items related to strategies that can enhance communication. Scoring for the CSS reflects how frequently a specific situation or behaviour occurs. The HADS comprises 14 items separated into two subscales: anxiety and depression. Higher scores reflect more symptoms of anxiety and depression.

The International Outcome Inventory for Hearing Aids $(\text { IOI-HA })^{32}$ includes seven questions measuring specific dimensions of HA outcomes: daily use, benefits, remaining activity limitations, satisfaction, remaining participation restrictions, impact on the environment and quality of life, with higher scores indicating better outcomes. The IOI-HA was not used as an outcome measure in this study; rather, it was used to describe the demographic and clinical characteristics of the participants, as shown in table 1 .

The HHIE, CSS and HADS were administered according to the methods described ${ }^{29-31}$ and were available on the study website, in Swedish. The questionnaires were administered online before and directly after the study participation and 6 months after participation to evaluate self-reported hearing problems, communication strategies and anxiety and depression. All of the questionnaires have a good internal consistency ${ }^{33} 34$ and have been shown to be as reliable as the original versions when used with a Swedish population of young adults and elderly. ${ }^{33}$ Thorén $e t a l^{55}$ stressed the importance of keeping the internet-based administration format of the HHIE and HADS stable across time points.

\section{Intervention group}

The internet-based intervention programme is based on four elements: reading, home training, interaction with an audiologist and interaction with peers in an internet-based discussion forum, as seen in figure 2. The participants received information about the intervention programme and access to the reading material on the study website; they also received a book about hearing and $\mathrm{HAs}^{36}$ and the Swedish version of Active Communication Education, a compendium of communication strategies. ${ }^{5378}$ The website information about the intervention programme, along with the book and the compendium, was also mailed to all of the participants in the intervention group.

The reading element is divided into five modules, one module for each of the 5 weeks. The participants were instructed to read specific content each week based on the various chapters of the book and information from the compendium. ${ }^{18}$ The weekly home assignments (weeks 1-5) were accessible to facilitate an understanding of the contents of the book and the compendium. For example, the weekly assignments could be to observe the benefits of using HAs. The weekly home assignments were handed in on the internet by the participants (weeks 1-5), and direct responses were provided online by an audiologist. The weekly home assignments were also discussed

\section{1 week \\ $\times 5$}

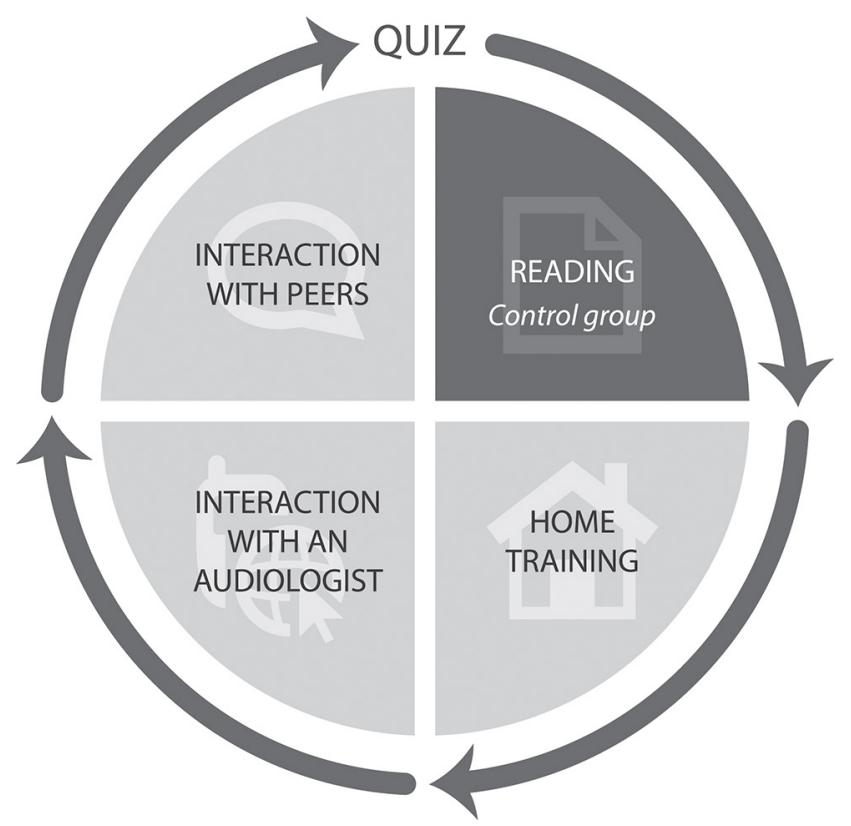

Figure 2 The full internet-based programme outlined for the intervention group and the element of the internet-based programme outlined for the control group. The full internetbased programme consisted of four elements: reading, home training, interaction with an audiologist and interaction with peers in an internet-based discussion forum. Weeks 1-4 of the intervention concluded with a quiz. The small part of the internet-based programme consisted of the reading element.

with the audiologist over the phone at the end of each treatment week. The telephone consultations lasted approximately $10-15$ min per participant and provided the participants with an opportunity to reflect on the assignment and discuss any concerns they might have. Weeks 1-4 ended with quiz questions on the content of the past weeks' readings. The participants in the intervention group were invited to attend a discussion forum on the study website. Weekly topics were presented for the participants ${ }^{18}$ to discuss with one other, without any interaction with the audiologist. The participants were free to use the discussion forum with no restrictions from the audiologist. However, all activities were strictly observed, and if needed, inappropriate postings could be deleted. No inappropriate postings occurred.

\section{Control group}

The control group received one reading element (see figure 2), that is, the first four chapters of the book, ${ }^{36}$ and the information about participation was provided on the study website. The website information and book chapters were also mailed to the participants. The control group was asked to read the four chapters over a 5-week period; no assignments were given in association with their participation. To minimise the impact of professional interaction, no monitoring was provided during 
the programme to ensure that the participants actually read the chapters.

\section{Follow-up}

At the end of the treatment period, the HHIE, CSS and HADS were made available to all participants on the study website, and the participants were asked to complete them. Both groups' participation was evaluated using a poststudy telephone interview. The poststudy interviews for the intervention group were conducted by five different clinical audiologists, compared with one who conducted the prestudy interviews and the telephone consultations during the study, to minimise the influence of special attention on the participants' responses to the questionnaires. The five audiologists were trained for consistency by the project leader. The poststudy interviews for the control group were conducted by the same audiologist who conducted the prestudy interviews. For the telephone interview, the audiologists used a self-designed form that contained questions about the study process, including opportunities for the participants to provide their own comments. Different forms were designed for the intervention group and the control group. All of the participants were invited to keep their copy of the reading material.

Six months after the study participation, the participants in both groups were contacted via e-mail and asked to complete the HHIE, CSS and HADS online again.

\section{Statistical analyses}

Statistical Package for the Social Sciences ${ }^{39}$ software for Windows (SPSS V.19.0) was used for the analysis of all data. Three measurement time points were examined: pretreatment (T0), post-treatment (T1) and 6 months post-treatment (T2). To ensure a between-group effect of $80 \%$ at the $5 \%$ significance level, it was estimated that 60 participants needed to be included in the study. An effect size of Cohen's $d=0.80$ was expected. The expected standardised mean difference on the HHIE total scale formed the basis of the obtained power. The within-group and between-group effect sizes of Cohen's $d$ were calculated from $\mathrm{T} 0$ to $\mathrm{T} 1$ and from $\mathrm{T} 0$ to $\mathrm{T} 2$, and were categorised as small $(0.2 \leq d<0.5)$, moderate $(0.5 \leq d<0.8)$ and large $(0.8 \leq d)$.

No significant differences were found between the groups at T0 for all the outcome measures. All data from the participants who did not complete $\mathrm{T} 1$ and/or T2 measurements were treated on an intention-to-treat (ITT) basis, ${ }^{40}$ meaning that the participants were included in the analysis (as missing data) regardless of their compliance or withdrawal from the study (see figure 1).

Given the ability to handle missing data, ${ }^{41}$ mixed-effects models with compound symmetry as the covariance structure were used to analyse the HHIE, CSS and HADS. Differences between the intervention group and the control group were examined by modelling the interaction effects of group and time. A subgroup analysis was performed including two groups categorised as age group 20-59 years and age group 60-80 years.

A sensitivity analysis was performed using mixed-effects models for the HHIE, CSS and HADS, this time excluding subjects who did not complete all measurement time points (T1 and/or T2). Sensitivity analysis was performed to increase the understanding of the relationships between internet-based AR and the outcome measures, HHIE, CSS and HADS.

\section{RESULTS}

\section{Attrition and adherence}

Eight participants in the intervention group and five in the control group completed the study programme but did not provide all $\mathrm{T} 1$ and/or T2 measurements, without giving a specific reason. Six participants in the intervention group and seven in the control group withdrew from participation in the study, as shown in figure 1. Five of those who withdrew from the study provided T1 measurements; four provided T2 measurements. One participant who was lost to follow-up at $\mathrm{T} 1$ provided $\mathrm{T} 2$ measurements. Consequently, 12 participants (16\%) did not provide $\mathrm{T} 1$ measurements (of which $\mathrm{n}=1$ followed up only with the HHIE), and 22 participants did not provide T2 measurements (30\%). No significant differences were found when comparing the baseline values between those who discontinued the study programme from T0 to T1 and those who did not. Those who discontinued from $\mathrm{T} 0$ to $\mathrm{T} 2$ had lower scores on baseline values for HHIE total $(\mathrm{t}(72)=-2.31, \mathrm{p}=0.024)$ and the emotional subscale $(\mathrm{t}(72)=-2.05, \mathrm{p}=0.044)$, and lower points on HADS total $(\mathrm{t}(72)=-2.73, \mathrm{p}=0.008)$ and the anxiety $(\mathrm{t}(72)=-2.03, \quad \mathrm{p}=0.046)$ and depression $(\mathrm{t}(72)=-2.38$, $\mathrm{p}=0.020)$ subscales, compared with those who continued with the study.

Thirteen per cent of the participants in the intervention group who completed the study programme answered less than three (of four) of the weekly quizzes, and $26 \%$ provided less than four (of five) online weekly responses to the audiologist. However, all of these were active participants in conversations during the weekly telephone follow-up, and some stated a wish for the discussion forum to be more active because they considered that part of the intervention very interesting. On average, the participants posted 0.4 contributions to the discussion forum.

\section{Primary outcome measure}

Both groups showed decreased HHIE total scores T0$\mathrm{T} 1 \quad(\mathrm{p}<0.000)$ and $\mathrm{T} 0-\mathrm{T} 2 \quad(\mathrm{p}<0.000)$. The interaction effect for HHIE total T0-T1/T0-T2 was not significant. The results are presented in table 2, and the estimated marginal means and SEs of the outcome measures HHIE, CSS and HADS for both groups are presented as online supplementary appendix I. Both groups showed decreased scores for both of the HHIE subscales from T0 to T1 $(\mathrm{p}<0.001)$ and from T0 to T2 $(\mathrm{p}<0.001)$. The 


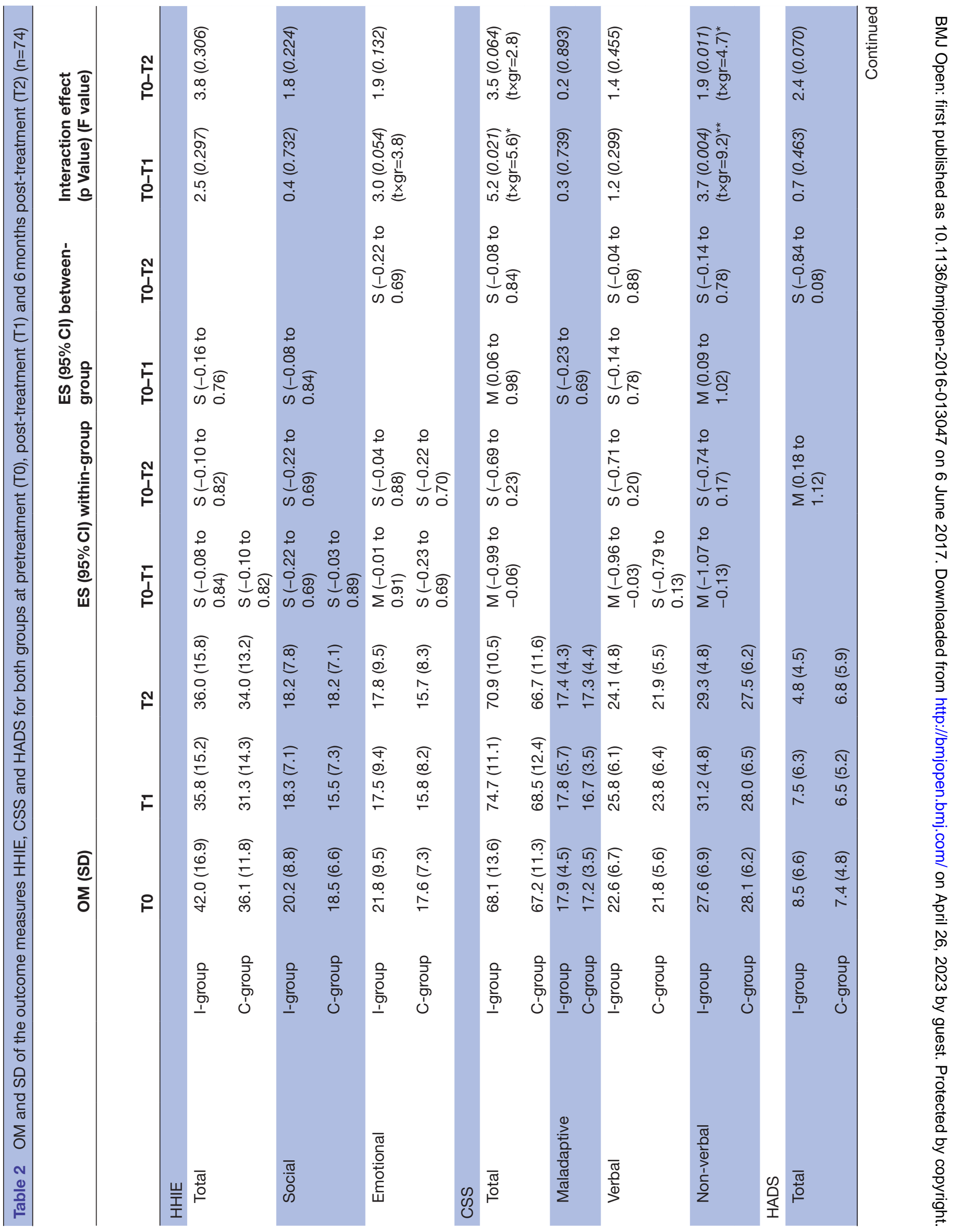




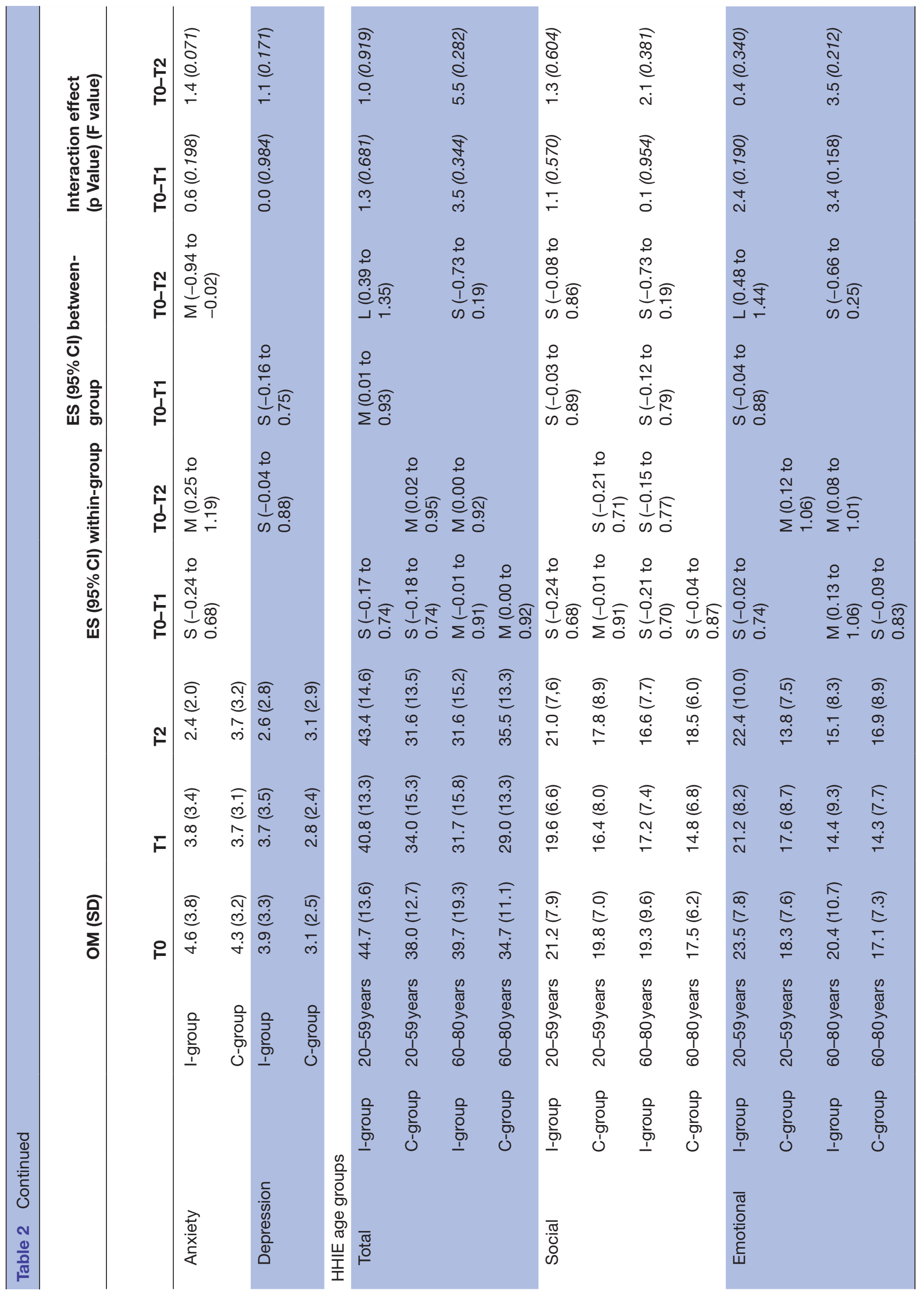




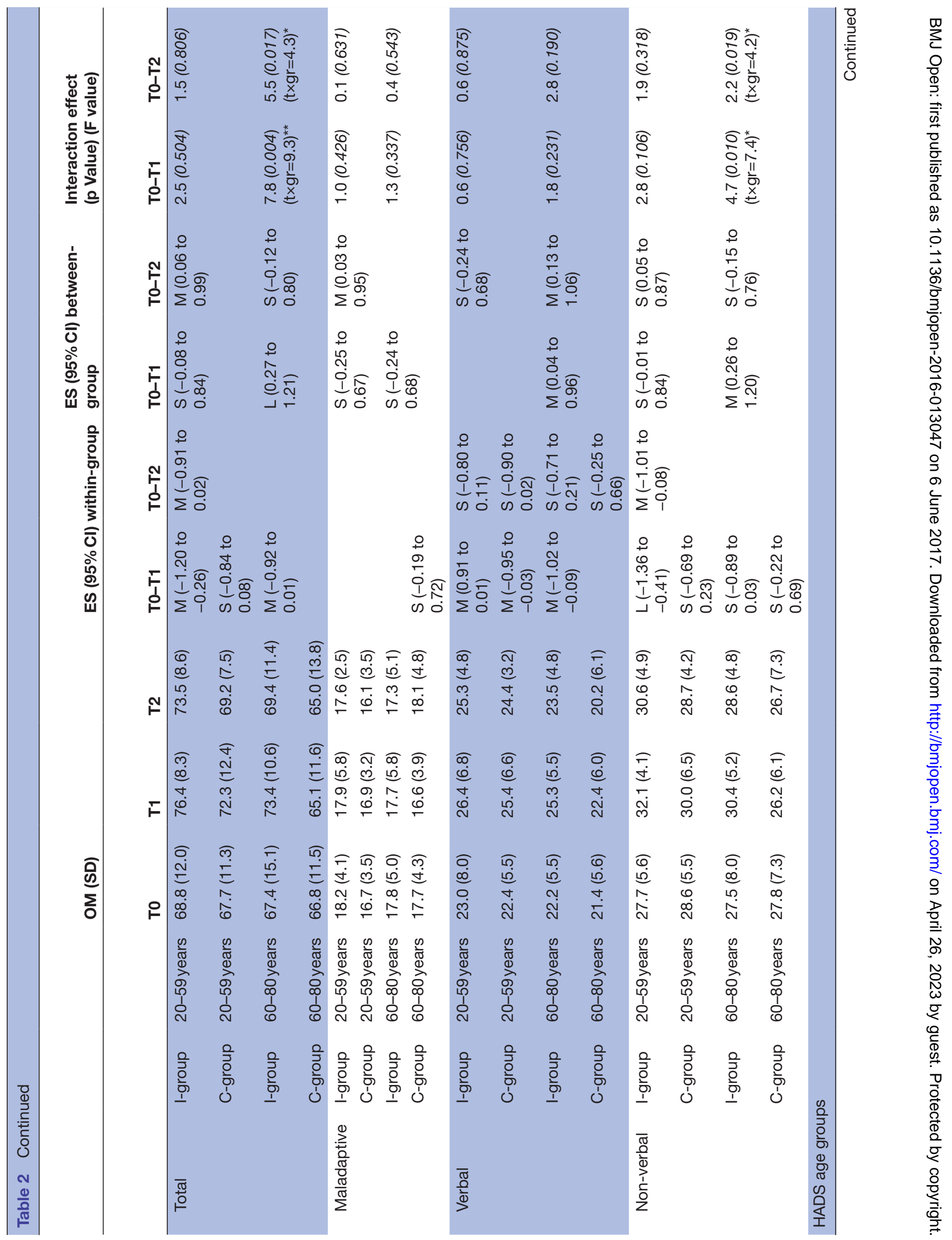




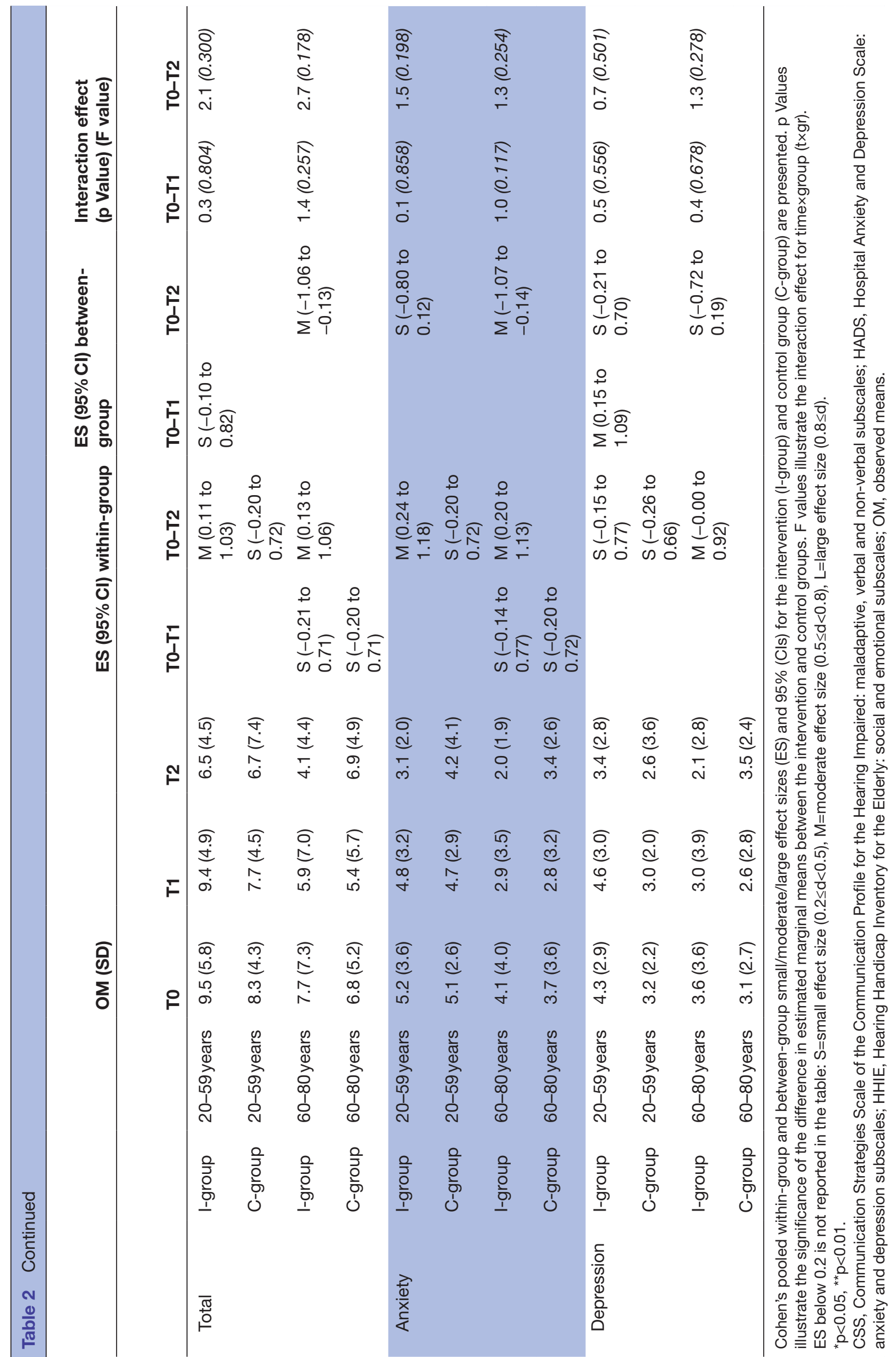

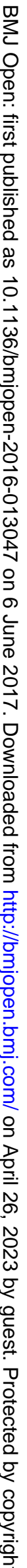


interaction effect was not significant for $\mathrm{T} 0-\mathrm{T} 1$ for the social subscale or for T0-T2 for the social and emotional subscales. A borderline significant interaction effect emerged for the emotional subscale $\mathrm{T} 0-\mathrm{T} 1 \quad\left(\mathrm{~F}_{(1,64.3)}=3.8\right.$, $\mathrm{p}=0.054)$. Small to large between-group effect sizes were found for the HHIE, as shown in table 2.

\section{Secondary outcome measures}

Significantly greater improvement was found for the intervention group compared with the control group from T0 to T1 for the CSS total $\left(\mathrm{F}_{(1,62.9)}=5.6, \mathrm{p}<0.05\right)$ and for the non-verbal subscale $\left(\mathrm{F}_{(1,63.8)}=9.2, \mathrm{p}<0.01\right)$. This interaction effect persisted from T0 to T2 for the non-verbal subscale $\left(\mathrm{F}_{(2,115.8)}=4.7, \mathrm{p}<0.05\right)$, and was on the borderline for CSS total $\left(\mathrm{F}_{(2,114.4)}=2.8, \mathrm{p}=0.064\right)$. Moderate within-group effect sizes from T0 to T1 were observed for the intervention group for the CSS total and for the verbal and non-verbal subscales. Moderate between-group effect sizes were shown for the CSS total and for the non-verbal subscale from $\mathrm{T} 0$ to $\mathrm{T} 1$, as shown in table 2.

The analyses for HADS showed that both the intervention group and the control group improved their total scores over the time, and the analyses identified no significant differences when modelling the interaction effects from T0 to T1 or from T0 to T2, as shown in table 2. Moderate within-group effect sizes were found for the HADS total from T0 to T2, as shown in table 2.

\section{Subgroup analysis}

A subgroup analysis for different age groups was performed for the HHIE, CSS and HADS scores from $\mathrm{T} 0$ to $\mathrm{T} 1$ and from T0 to T2. No significant interaction effect was found for the outcome measures HHIE and HADS from T0 to T1 or from T0 to T2 for the age groups 20-59years and 60-80years, as shown in table 2. Nevertheless, a large between-group effect was found from T0 to T2 for the HHIE total score and for the emotional subscale among the participants in the 20-59years age group, as shown in table 2. The participants in the 60-80years age group showed medium between-group effect sizes for the HADS total scale and anxiety subscale.

The CSS total showed an interaction effect from T0 to T1 $\left(\mathrm{F}_{(1,33.2)}=9.3, \mathrm{p}<0.01\right)$, indicating that the 60 -yearolds to 80-year-olds in the intervention group showed significantly more improvement than the 60 -year-olds to 80 -year-olds in the control group. This effect persisted from $\mathrm{T} 0$ to $\mathrm{T} 2\left(\mathrm{~F}_{(2,63.7)}=4.3, \mathrm{p}<0.05\right)$. There was also an interaction effect from T0 to T1 for the non-verbal subscale, with the 60-year-olds to 80-year-olds in the intervention group showing significantly greater improvement $\left(\mathrm{F}_{(1,33.7)}=7.4\right.$, $\mathrm{p}<0.05)$ compared with the 60 -year-olds to 80 -year-olds in the control group. This effect persisted from T0 to T2 $\left(\mathrm{F}_{(2,64.1)}=4.2, \mathrm{p}<0.05\right)$. It may be noted that the participants of the age 60-80 years in the control group declined over time as measured by CSS total and non-verbal subscale, and that improvements in the intervention group were of small or moderate effect size. However, the younger subgroup (20-59 years of age) was improving over time in both the control (small effect sizes) and the intervention group (moderate or large effect sizes).

\section{Sensitivity analysis}

A sensitivity analysis was performed for the HHIE, CSS and HADS by excluding all data from the participants who did not complete all three measurement time points (T0, T1 and T2; $\mathrm{n}=50$ ). However, 50 participants are not sufficient to ensure a between-group effect of $80 \%$. Nonetheless, the sensitivity analysis revealed an interaction effect for the HHIE emotional subscale from T0 to T1 ( $\mathrm{F}_{(1,}$ ${ }_{48.0)}=4.3, \mathrm{p}<0.05$ ), with the intervention group showing an advantage, as shown in online supplementary appendix II. This interaction effect did not remain 6 months post-treatment.

The interaction effect for the CSS total that was achieved when participants were treated on an ITT basis $(\mathrm{n}=74)$ was not apparent in the sensitivity analysis $(\mathrm{n}=50)$. The results for the CSS showed an interaction effect for the non-verbal subscale from $\mathrm{T} 0$ to $\mathrm{T} 1\left(\mathrm{~F}_{(1,48.0)}=6.8, \mathrm{p}<0.05\right)$ and from T0 to T2 $\left(\mathrm{F}_{(2,96.0)}=3.5, \mathrm{p}<0.05\right)$, with the intervention group showing significantly greater improvement compared with the control group, similar to the results for the whole group $(n=74)$.

Furthermore, the sensitivity analysis showed significant results for the HADS total scale from T0 to T2 $\left(\mathrm{F}_{(2,96.0)}=3.1\right.$, $\mathrm{p}<0.05$ ), indicating that the intervention group's scores had improved more than those of the control group. The sensitivity analysis for the remaining scales and subscales showed no changes in significance compared with the previous analysis $(\mathrm{n}=74)$, as shown in online supplementary appendix II.

\section{DISCUSSION}

The aim of this study was to evaluate whether internet-based AR for HA users would be effective in GCP and whether the assumed positive effect of participating in the internet-based AR programme would be maintained 6 months after the programme was completed. Our aim was also to analyse the effect of the programme in two age groups.

Both the intervention group and the control group improved their HHIE scores from $\mathrm{T} 0$ to $\mathrm{T} 1$ and from T0 to T2; however, the improvements were not significantly different between groups, unlike the findings of our research group's previous study ${ }^{18}$ (for demographics, see table 1). Differences in the results could be related to differences in the recruitment process. In our previous study, ${ }^{18}$ the participants were recruited through advertisements and articles in Swedish national daily newspapers and on the internet; the recruited participants were well educated and had a higher education level than the general population. This indicated that the intervention programme is well suited for educated patients, although education was not a significant predictor of intervention outcomes. ${ }^{42}$ This recruitment strategy recruited participants who actively sought involvement in research. 
Additionally, the participants were somewhat older and were more experienced HA users than the participants in the current study. Thus, the internet-based intervention programme may be more suited for older adults and experienced HA users than for younger adults and less experienced. Also, the participants in the current study received similar clinical treatments prior to participating in the study, which may impact the effectiveness of the current study, in particular if the participants experience positive clinical treatment outcomes. Another underlying explanation for the differences in improvement could be that the control group was more active in the present study compared with our previous study, ${ }^{18}$ in which the participants read a book about the history of HAs, although not online. Participants being enrolled in a research study might generally be more positive after their participation, ${ }^{43}$ which could be considered a research bias assuming that the full internet-based AR is more effective than one element of the programme. A borderline significant interaction effect emerged for the HHIE emotional subscale from $\mathrm{T} 0$ to $\mathrm{T} 1$, indicating that the full internet-based AR could have had a positive impact on the emotional effects of the participants' hearing loss. This could be due to the reading and home training elements that by educating raises participants' abilities, which can lead to increased self-esteem. This increased self-esteem might be additionally acknowledged by the audiologist during the weekly telephone consultations.

The sensitivity analysis that was performed $(n=50)$ revealed an interaction effect for the HHIE emotional subscale from T0 to T1, for the CSS non-verbal strategies subscale from $\mathrm{T} 0$ to $\mathrm{T} 1$ and from $\mathrm{T} 0$ to $\mathrm{T} 2$, and for the HADS total scale from $\mathrm{T} 0$ to $\mathrm{T} 2$; all interaction effects indicated an advantage for the intervention group. It appears that participants who were especially persistent and who participated in all aspects of the full internet-based AR or were just conscientious may show changes in the HHIE score and in their communications skills, and changes in symptoms of anxiety and depression. Thus, the sensitivity analysis makes the study underpowered, and these interaction effects should be treated with caution.

The participants who did not provide T0-T2 measurements for the present study had lower baseline scores on the HHIE and HADS compared with those who continued in the present study, indicating that insubstantial self-reported social and emotional effects of hearing loss as well as anxiety and depression symptoms can influence the decision to drop out. Laplante-Lévesque $e t a t^{42}$ showed that greater self-reported hearing disability is one of the predictors for intervention uptake and positive outcomes. Another potential influencing factor might be that it is easier to drop out when the intervention is internet-based, as discussed by Andersson et al. ${ }^{44}$

In our earlier research, the HHIE was an appropriate measure for the outcomes of telephone-supported AR beyond HA fitting in $\mathrm{GCP}^{17}$; in that study, the programme for the intervention group did not include parts of the Active Communication Education (ACE) programme, which targets the communication difficulties experienced by older people with hearing impairment in everyday life. ${ }^{37}$ In the clinical population of the present study, we found effects for the CSS total and the non-verbal subscale; thus, it seems that participating in the full internet-based AR programme containing the ACE programme has a larger effect on communication skills compared with partial participation. Determining the element responsible for the interaction improvement in the present study is challenging. The reading and home training elements of the full internet-based AR programme might have contributed to improved communication skills, but so, too, might the telephone follow-up by the audiologist. Having personal phone contact with an audiologist may have encouraged the participants to try out the programme's suggested strategies. The effect on the CSS, however, raises doubts about the applicability of the HHIE as a main outcome measure for the present study (eg, power calculation and sample size).

The intervention and control groups were also analysed using subgroups. The 60-year-olds to 80 -year-olds in the intervention group obtained significantly greater improvement compared with the 60-year-olds to 80 -yearolds in the control group in terms of the CSS total and the non-verbal subscale, contradicting our hypothesis that the 20-59years age group would be more receptive to internet-based AR. As mentioned, the ACE programme targets the everyday life of older people, which may have been reflected in the results of the CSS subgroup analysis. However, it might be that the decline in scores as measured by CSS total and the non-verbal subscale for the 60-year-olds to 80-year-olds in the control group contributes to the small effect in the intervention group becoming more pronounced in this subgroup than the differences in the improvements seen in both control and intervention groups in the younger subgroup (20-59 years). Additionally, it might be that the older adults use more non-verbal strategies when communicating because of their presumably greater cognitive demands when trying to understand speech. ${ }^{45}$ However, the subgroup analysis includes small groups and these results should be treated with caution.

Thorén $e t a l^{18}$ found significant improvements in the intervention group when measuring participants' psychosocial well-being using the HADS. Our results showed that both the intervention group and the control group showed improved HADS scores, although the difference between the groups was not significant. Preminger ${ }^{46}$ reviewed the importance of taking psychosocial outcomes into account when implementing group adult AR and highlighted the importance of outcome studies. The HADS is believed to be sensitive enough to detect the effects of online education. ${ }^{1618}$

\section{Limitations}

One limitation is that the participants in this study have been HA users for an average of 7.5 years. In our previous study in a GCP setting, that number was 6.5 years ${ }^{17}$; for 
Thorén $e t$ al, the average was 9.9 years. ${ }^{18}$ Despite inclusion criteria that acknowledged the heterogeneity of a clinical population, the participants in the present study were experienced HA users. However, new HA users are more likely to benefit from educational programmes, compared with experienced HA users. ${ }^{13}$ Thus, although different aspects of AR may not suit every individual client, the current study increases the confidence that the clinical use of group AR will likely have positive outcomes. ${ }^{47}$ Additionally, included participants were asked to sign up via the internet and are thus competent internet users, which would likely impact the effectiveness of the current study. Another limitation is that the control group received an active intervention and only one of the four elements of the programme, as seen in figure 2. We are unaware of the relative benefit that any other element alone/combination of elements might have as compared with the full intervention. A more clear result may generate from a control group that receives no intervention.

Another concern that needs to be mentioned is that the observed standardised mean difference on the HHIE between the intervention and control group was much lower than what was expected when comparing with previous research and anticipated in the sample size calculation; also, the SD was larger than expected. Thus, increasing the sample size initially could maybe result in statistically significant difference between the groups; nevertheless, it is not certain that the standardised mean difference between the groups in a larger sample would lead to clinically meaningful difference for the participants.

\section{CONCLUSION}

The internet-based approach expands the availability of AR in GCP, offering accessibility to many people, including hard-to-reach populations. ${ }^{48}$ The present study shows that using the internet for interactions between the audiologist and the HA user had a positive effect on communication skills for the intervention group compared with the control group. Furthermore, the full internet-based AR programme was not more effective than one element of the internet-based AR programme. However, the advantages of an internet-based approach, both for the patient and the clinician, ${ }^{47}$ may inspire clinicians and operation managers in their future utilisation of comprehensive AR in addition to HA fitting.

More research is needed to examine the efficacy and applicability of this type of intervention. This study is one of the first RCTs in Sweden to implement internet-based rehabilitation beyond conventional HA fitting in a GCP, and is at the beginning of exploring the possible clinical applicability of this type of intervention. Further analysis is needed to examine the individual elements of the full internet-based AR programme to evaluate which part of the internet-supported educational intervention had the greatest effect: the reading material, the weekly assignments, the discussion forum or the contact with the audiologist. In addition, guided internet-based intervention should be compared with face-to-face $\mathrm{AR}$ to analyse whether the two approaches are equally effective. Also, this type of internet-based intervention delivered exclusively to new HA users should be compared with a matched group who only receive HA in order to know the relative efficacy of the internet-based AR programme. Additionally, the individual needs of the HA user should be taken into account when designing group $\mathrm{AR}$, as should including significant others in the intervention.

Acknowledgements The authors would like to thank the clinicians and operation managers at the Hearing Organization at Södra Älvsborg, Sweden, for their financial and administrative support. We are also grateful to all the participants, and to Alexander Alasjö and George Vlaescu for helping with the websites included in the intervention portal. A report of the trial was presented at the First International Meeting on the Internet and Audiology 3-4 October 2014, Linköping, Sweden.

Contributors GA, TL and MM contributed to the conception and design of the study, and to the acquisition of data for the study. MM contributed to data collection and analysed the data. GA, TL, MM and KK contributed to the analysis of data and participated in interpretation of data for the study, in drafting the study and revising it critically for important intellectual content, and in giving final approval of the version to be published. GA, TL and KK provided continuous supervision during the entire study.

Funding This work was sponsored by the Stinger Foundation; Oticon Foundation; Habilitation and Health, Region Västra Götaland, Research and Technology Development; and in part by a programme grant from the Swedish Council for Wealth, Working Life and Welfare (FORTE, 2009-0055).

Competing interests None declared.

Ethics approval The regional ethical review board in Gothenburg, Sweden.

Provenance and peer review Not commissioned; externally peer reviewed.

Open Access This is an Open Access article distributed in accordance with the Creative Commons Attribution Non Commercial (CC BY-NC 4.0) license, which permits others to distribute, remix, adapt, build upon this work non-commercially, and license their derivative works on different terms, provided the original work is properly cited and the use is non-commercial. See: http://creativecommons.org/ licenses/by-nc/4.0/

(c) Article author(s) (or their employer(s) unless otherwise stated in the text of the article) 2017. All rights reserved. No commercial use is permitted unless otherwise expressly granted.

\section{REFERENCES}

1. World Health Organization. International classification of functioning, disability and Health (ICF. Geneva: World Health Organization, 2001.

2. Boothroyd A. Adult aural rehabilitation: what is it and does it work? Trends Amplif 2007;11:63-71.

3. Hickson L, Worrall L. Beyond hearing aid fitting: improving communication for older adults. Int J Audiol 2003;42:84-91.

4. Hawkins DB. Effectiveness of counseling-based adult group aural rehabilitation programs: a systematic review of the evidence. J Am Acad Audiol 2005;16:485-93.

5. Hickson L, Worrall L, Scarinci N. Measuring outcomes of a communication program for older people with hearing impairment using the International Outcome Inventory. Int J Audiol 2006;45:238-46.

6. Chisolm T, Arnold M. Evidence about the effectiveness of aural rehabilitation programs for adults. In: Wong L, Hickson L, eds. Evidence-based practice in audiology: evaluating interventions for children and adults with hearing impairment. San Diego, CA: Plural, 2012:237-66.

7. Abrams H, Chisolm TH, McArdle R, Hnath-Chisolm T. A cost-utility analysis of adult group audiologic rehabilitation: are the benefits worth the cost? J Rehabil Res Dev 2002;39:549-58.

8. Kochkin S. Marke Trak VIII: 25-years in trends in the hearing health market. Hearing review 2009;16:12-31. 
9. Grenness C, Hickson L, Laplante-Lévesque A, et al. Patientcentred care: a review for rehabilitative audiologists. Int J Audiol 2014;53:S60-S67.

10. McCormack A, Fortnum $\mathrm{H}$. Why do people fitted with hearing aids not wear them? Int J Audiol 2013;52:360-8.

11. Knudsen LV, Oberg M, Nielsen C, et al. Factors influencing help seeking, hearing aid uptake, hearing aid use and satisfaction with hearing aids: a review of the literature. Trends Amplif 2010;14:127-54

12. Laplante-Lévesque A, Hickson L, Worrall L. Rehabilitation of older adults with hearing impairment: a critical review. J Aging Health 2010;22:143-53.

13. Kramer SE, Allessie GH, Dondorp AW, et al. A home education program for older adults with hearing impairment and their significant others: a randomized trial evaluating short- and long-term effects. Int $J$ Audiol 2005;44:255-64.

14. Laplante-Lévesque A, Pichora-Fuller MK, Gagné JP. Providing an internet-based audiological counselling programme to new hearing aid users: a qualitative study. Int J Audiol 2006;45:697-706.

15. Sweetow RW, Sabes JH, Henderson SJ. Technologic advances in aural rehabilitation: applications and innovative methods of service delivery. Trends Amplif 2007;11:101-11.

16. Thorén $E$, Svensson $M$, Törnqvist $A$, et al. Rehabilitative online education versus internet discussion group for hearing aid users: a randomized controlled trial. J Am Acad Audiol 2011;22:274-85.

17. Lundberg $M$, Andersson $G$, Lunner $T$, A randomized LT. A randomized, controlled trial of the short-term effects of complementing an educational program for hearing aid users with telephone consultations. J Am Acad Audiol 2011;22:654-62.

18. Thorén $\mathrm{ES}$, Oberg $\mathrm{M}$, Wänström $\mathrm{G}$, et al. A randomized controlled trial evaluating the effects of online rehabilitative intervention for adult hearing-aid users. Int J Audiol 2014;53:452-61.

19. Ferguson $M$, Brandreth $M$, Brassington W, et al. A Randomized Controlled Trial to evaluate the benefits of a Multimedia Educational Program for First-Time Hearing Aid Users. Ear Hear 2016;37:123-36.

20. Molini-Avejonas DR, Rondon-Melo S, Amato CA, et al. A systematic review of the use of telehealth in speech, language and hearing sciences. J Telemed Telecare 2015;21:367-76.

21. Barak A, Sadovsky Y. Internet use and personal empowerment of hearing-impaired adolescents. Comput Human Behav 2008;24:1802-15.

22. Henshaw $\mathrm{H}$, Clark DP, Kang $\mathrm{S}$, et al. Computer skills and internet use in adults aged 50-74 years: influence of hearing difficulties. $J$ Med Internet Res 2012;14:e113.

23. Thorén ES, Oberg $M$, Wänström G, et al. Internet access and use in adults with hearing loss. J Med Internet Res 2013;15:e91.

24. Kaldo-Sandström V, Larsen HC, Andersson G. Internetbased cognitive-behavioral self-help treatment of tinnitus: clinical effectiveness and predictors of outcome. Am J Audiol 2004:13:185-92.

25. Hedman E, Ljótsson B, Rück $\mathrm{C}$, et al. Effectiveness of internet-based cognitive behaviour therapy for panic disorder in routine psychiatric care. Acta Psychiatr Scand 2013;128:457-67.

26. Moore AN, Rothpletz AM, Preminger JE. The effect of chronological age on the Acceptance of Internet-Based Hearing Health Care. Am J Audiol 2015;24:280-3.

27. Hopewell S, Clarke M, Moher D, et al. CONSORT for reporting randomized controlled trials in journal and conference abstracts: explanation and elaboration. PLoS Med 2008;5:e20.

28. Moher D, Hopewell S, Schulz KF, et al. CONSORT 2010 explanation and elaboration: updated guidelines for reporting parallel group randomised trials. Int J Surg 2012;10:28-55.
29. Ventry IM, Weinstein BE. The hearing handicap inventory for the elderly: a new tool. Ear Hear 1982;3:128-34.

30. Demorest ME, Erdman SA. Development of the communication profile for the hearing impaired. J Speech Hear Disord 1987;52:129-43.

31. Zigmond AS, Snaith RP. The hospital anxiety and depression scale. Acta Psychiatr Scand 1983;67:361-70.

32. Cox R, Hyde M, Gatehouse S, et al. Optimal outcome measures, research priorities, and international cooperation. Ear Hear 2000;21:106-15.

33. Öberg M, Lunner T, Andersson G. Psychometric evaluation of hearing specific self-report measures and their associations with psychosocial and demographic variables. Audiol Med 2007:5:188-99.

34. Hallberg LR, Eriksson-Mangold M, Carlsson SG. Psychometric evaluation of a swedish version of the communication strategies scale of the communication profile for the hearing impaired. $J$ Speech Hear Res 1992;35:666-74.

35. Thorén ES, Andersson G, Lunner T. The use of research questionnaires with hearing impaired adults: online vs. paper-andpencil administration. BMC Ear Nose Throat Disord 2012;12:12:12.

36. Elberling C, Worsøe K. When the sounds are weaker - on hearing and hearing aids. Herlev: Bording A/S, 2006.

37. Hickson L, Worrall L, Scarinci N. A randomized controlled trial evaluating the active communication education program for older people with hearing impairment. Ear Hear 2007;28:212-30.

38. Oberg M, Bohn T, Larsson U, Short- LU. Short- and long-term effects of the modified swedish version of the active communication education (ACE) program for adults with hearing loss. J Am Acad Audiol 2014;25:848-58.

39. SPSS (data analysis software system). Statistical Package for the Social Sciences for Windows. Version 19.0 www.spss.com 2011.

40. Fergusson D, Aaron SD, Guyatt G, et al. Post-randomisation exclusions: the intention to treat principle and excluding patients from analysis. BMJ 2002;325:652-4.

41. Gueorguieva R, Krystal JH, Move Over A. Move over ANOVA: progress in analyzing repeated-measures data and its reflection in papers published in the archives of General Psychiatry. Arch Gen Psychiatry 2004;61:310-7.

42. Laplante-Lévesque A, Hickson L, Worall L. What makes adults with hearing impairment take up hearing aids or communication programs and achieve successful outcomes? Ear Hear 2011;32:1-15.

43. Linde K, Fässler M, Meissner K. Placebo interventions, placebo effects and clinical practice. Philos Trans R Soc Lond B Biol Sci 2011;366:1905-12

44. Andersson G, Strömgren T, Ström L, et al. Randomized controlled trial of internet-based cognitive behavior therapy for distress associated with tinnitus. Psychosom Med 2002;64:810-6.

45. Pickora-Fuller MK. Processing speed and timing in aging adults: psychoacoustics, speech perception, and comprehension. Int $J$ Audiol 2003;42:59-67.

46. Preminger JE. Issues associated with the measurement of psychosocial benefits of group audiologic rehabilitation programs. Trends Amplif 2007;11:113-23.

47. Malmberg M, Lunner T, Kähäri K, et al. Implementing Internet-Based Aural Rehabilitation in a General clinical practice. Am J Audiol 2015;24:325-8.

48. Swanepoel deW, Hall JW. A systematic review of telehealth applications in audiology. Telemed J E Health 2010;16:181-200. 